\title{
Nostalgie, temps et espace dans La maison de Shemiran de Goli Taraghi
}

\author{
Esfaindyar Daneshvar
}

Published online: 15 December 2011

(C) The Author(s) 2011. This article is published with open access at Springerlink.com

\begin{abstract}
After the Islamic Revolution of Iran in 1979, a wave of political refugees and intellectuals immigrated to France. Among the intelligentsia, writers continued to write despite the practical and emotional difficulties of exile. Three decades later, some authors, such as Goli Taraghi, gradually developed a FrenchPersian hybrid literature, which closely interweaves the history and culture of both countries. Their stories put in perspective a deep understanding of time and space, past and present in terms of nostalgia and melancholy of the early years. In $\mathrm{La}$ maison de Shemiran, Goli Taraghi reveals a painful, intimate and sometimes humorous period of her life in exile and her childhood memories. Her story is the a posteriori result of this experience, which however deeply penetrates the psychological mechanism that operates the inner self as a mutative agent of identitary metamorphosis. By studying this work, we will take a look at the intrinsic relationship between nostalgia caused by the situation of exile and its impact on the perception of time and space. We are dealing here with an individual experience, which nevertheless reflects a painful and transitional phase, also known by other artists in exile.
\end{abstract}

Keywords Nostalgia · French-Persian literature - Goli Taraghi ·

Exile $\cdot$ Bergson $\cdot$ Time $\cdot$ Space

Dans le cadre de la recherche du transculturalisme qui caractérise un aspect important de la littérature franco-persane de ces dernières décennies, on peut risquer une catégorisation des écrivains en classifiant leur statut d'exilé (immigré ou réfugié politique) et leurs œuvres. Le genre et le style des œuvres, la langue de l'écriture

E. Daneshvar $(\bowtie)$

French Department, Leiden University, Leiden, The Netherlands

e-mail: e.daneshvar.tehranizadeh@hum.leidenuniv.nl 
(directement en français ou traduite), les aspects interculturels ou transculturels des œuvres sont en effet autant de paramètres relevants. Cette démarche nous permet de mieux situer l'œuvre de Goli Taraghi (1939), l'une des écrivaines franco-iraniennes les plus lues. L'auteur immigre en France en 1979, depuis elle voyage continuellement entre la France, l'Iran et les Etats-Unis. L'auteur écrit toujours en persan et veille méticuleusement à la traduction de ses œuvres, publiées en France et en Iran. Avec un style ironique et poétique, elle porte un regard critique sur les cultures et les sociétés qu'elle connaît. L'histoire et la psychologie occupent une place importante dans ses œuvres à la fois modernes et postmodernes.

La maison de Shemiran (2003) occupe une place spécifique dans l'œuvre de Taraghi. En effet, l'intérêt particulier de ce roman se trouve dans le dialogique culturel qu'il établit avec le pays d'accueil. L'œuvre étant en grande partie autobiographique selon l'auteur, ${ }^{1}$ exprime l'état nostalgique et dépressif du personnage dans les premières années d'exil en France. La narratrice est plongée dans ses souvenirs d'enfance tandis qu'elle passe un séjour dans une maison de repos psychiatrique. Sa pensée l'emporte vers le passé, alors qu'elle se bat pour sortir de la dépression avec l'écriture. La mutation de la nostalgie dans le temps est déterminante pour la possibilité d'une vie future. Transcender la nostalgie mélancolique permet à la narratrice de tendre vers une écriture créatrice et un dialogue avec le pays d'accueil. Finalement elle sort guérie de l'hôpital et envisage d'écrire l'histoire de cette transcendance vers une nouvelle phase d'intégration et de dialogue interculturelle.

Notre analyse portera plus particulièrement sur le thème de la nostalgie et son évolution dans cette œuvre. Bien que la nostalgie constitue un thème littéraire en soi, nous l'étudierons dans le cadre spécifique de l'exil. L'exil, en tant qu'arrachement au pays natal, s'accompagne souvent de mal du pays. De ce fait, le regard nostalgique de la protagoniste dépressive se tourne vers son passé, et sa perception spatio-temporelle en sera profondément affectée. Au début du roman, la narratrice revit son enfance en Iran non pas comme une remémoration d'un passé révolu, mais par de profondes vagues émotionnelles, balayant toutes distances spatio-temporelles conscientes entre le passé et le présent. Cette perception du temps rappelle fortement la notion bergsonienne de la durée, qui est étroitement liée à la création artistique et la notion de liberté. Or, la nostalgie dépressive semble mal s'accorder à ces concepts. Malgré cela la narratrice écrit et persiste dans la création. Quelle est l'influence exercée par la nostalgie sur la durée (comme perception créatrice)? La nostalgie est-elle un état rigide et figé ou passible de mutation? Quel est le lien entre la nostalgie et l'écriture chez la protagoniste?

L'analyse des temps et des instances narratifs clarifiera d'abord des liens et des impacts entre la nostalgie et la perception temporelle de la narratrice en rapport avec l'idée de durée. Ensuite, l'espace du pays natal et des éléments qui le symbolisent se présente également comme une toile de fond culturelle où évolue la nostalgie. Nous verrons enfin la portée de la lutte de la narratrice contre la nostalgie et l'exil, à travers la perspective d'une créativité en durée.

\footnotetext{
${ }^{1}$ Entretien personnel avec G. Taraghi, où elle affirme: « La plupart de mes histoires viennent de mes propres expériences. » Décembre 2009, Paris.
} 


\section{La nostalgie destructive et réparatrice}

Rappelons que l'étymologie du mot nostalgie est un néologisme français composé de deux mots grecs: nostos qui signifie « retour », et algos qui veut dire « souffrance ». Bolzinger (2007), il serait utilisé en 1803 pour la première fois dans ce sens par un étudiant de médecine (Jean Hofer) en Alsace, qui l'étudie chez les jeunes appelés à défendre le territoire national. Ce concept est souvent associé à l'éloignement douloureux du pays en 1793. Le sujet nostalgique « espère et croit pouvoir récupérer l'objet perdu, se rapprocher de l'objet éloigné » explique Moser (1999, p. 88). Au début du récit, la protagoniste nostalgique est fortement mélancolique, ${ }^{2}$ elle est exilée en France. L'objet perdu est non seulement le pays natal, mais aussi son identité. La nostalgie, thème fréquent et commun chez les écrivains iraniens exilés (Ghassemi, Javann, Saedi), est analysé par Karimi Hakkak. ${ }^{3}$ L'analyste définit deux caractéristiques distinctes appartenant à deux phases consécutives de la nostalgie en exil: destructive et réparatrice. Évidemment le dépassement de la première nostalgie s'opère chez certains artistes avec « difficulté, travail et discipline » selon l'analyste, et il ne s'agit nullement d'une évolution automatique. La destruction par la nostalgie menace l'exilé mentalement et physiquement. Aussi s'agit-il de voir dans ce récit, des effets de l'évolution de la nostalgie sur l'identité, donc la perception culturelle de l'exilée reflétée à travers ses écrits.

Dans les écrits des premières années d'exil, la nostalgie destructive est caractérisée par l'idéalisation du pays natal parallèlement à la dépréciation et au refus du pays d'accueil. Ensuite, dans la deuxième phase, si le «deuil » du passé s'effectue, émerge l'acceptation du présent de l'exil et du pays d'accueil. Un enrichissement culturel s'ajoute alors à l'activité créatrice de l'écrivain. Hakkak définit ainsi la nostalgie réparatrice:

L'aptitude de l'artiste exilé à prendre avec lui quelques gouttes essentielles de la culture de son pays, et de son sens profond; de les transformer ensuite dans son imaginaire et sa subjectivité, et d'offrir enfin aux lecteurs et à lui-même une nouvelle perspective de la vie (Ibid.).

Arrivée à cette phase, la nostalgie réparatrice n'est donc pas une déculturation ou une assimilation, mais plutôt la genèse d'un état d'hybridation culturelle. Cette «nostalgie » se rapprocherait plus de l'idée de «transculturation », qui, selon l'anthropologue cubain Ortiz (2002), est le processus par lequel une communauté emprunte certains matériaux à la culture majoritaire pour se les approprier et les

\footnotetext{
${ }^{2}$ La définition de la mélancolie, donnée par Julia Kristeva, reflète bien l'état psychologique de la narratrice: « ... c'est une affection grave qui se manifeste par un ralentissement psychique, idéatoire et moteur, par une extinction du goût pour la vie, du désir et de la parole, par l'arrêt de toute activité et par l'attrait irrésistible du suicide. » Dominique Grisoni, « Les abîmes de l'âme », Magazine littéraire, 244, 1987, p. 16.

3 Conférence au Kanoone Iranian, « À propos de Shahrokh Meskoob », USA 24/04/2006 (La conférence étant en persan, j'ai traduit des passages en français. Karimi Hakkak évoque la nostalgie « restorative » que je traduirais par « réparatrice »). Vidéo disponible sur: http://video.google.com/videoplay?docid= -2797947087284093082\#.
} 
refaçonner à son propre usage. Aussi, au début du récit, la langue et la culture française majoritaires sont d'emblée refusées par la protagoniste en mal du pays:

Je veux ouvrir la portière et m'échapper; reculer dans le temps, rentrer chez moi, loin de ce ciel étranger, de ce monde inconnu, de cette langue fugitive et inaccessible (p. 9).

À la fin du récit, elle montre une soif et joie de vivre dans ce nouveau pays:

Derrière les murs gris de ce parc, un autre monde m'attend. Un monde éveillé, avec l'odeur des cafés, les rues, les hommes, les paroles, les palpitations amoureuses, les promesses colorées, les sentiments délicieux, le soleil et les amis (p. 200).

K. Hakkak évoque les notions de «patrie » et « le sens de la patrie » comme lieu et lien de conflits psychiques et culturels en exil. Des lieux où se met en place tout un réseau de signes culturels. Le passage de la nostalgie destructrice vers la nostalgie réparatrice suppose donc une mutation et une transcendance de la culture initiale pour adapter un nouvel espace culturel. La perception temporelle reste au fond le paramètre central de la transcendance du personnage (le dépassement de la nostalgie destructrice).

Observons d'abord la nostalgie destructrice. Celle-ci se caractérise par trois phénomènes qui seront illustrés plus loin par des exemples:

- Un regard passéiste niant le présent du pays d'accueil.

- Une idéalisation du passé (le climat, la famille, le pays...).

- Une crise identitaire, ou un dédoublement schizophrénique.

Le moi identitaire de la narratrice se fracture dans le cadre d'exil, il se trouve à la fois ici et ailleurs (dans un passé révolu). La perception spatio-temporelle se modifie. Ces trois phénomènes se voient liés à la notion du temps et sa perception subjective. Or la perception temporelle de la narratrice (surtout au début du récit) semble chronologiquement continue et indivisible. Cette perception temporelle ressemble à l'idée de la durée que nous analyserons plus loin. Quelle est cette durée et comment est-elle exprimée à travers les temps de la narration?

\section{Les temps de la narration et la «durée »}

La narration du roman se fait en deux temps: le temps du récit raconté au présent de l'indicatif (la narratrice à la clinique psychiatrique), et le temps de l'histoire au prétérit (les souvenirs de l'enfance). Il semble s'agir dans un premier moment d'une narratrice intradiégétique procédant à une narration intercalée, car les souvenirs racontés par la narratrice sont aussi exprimés au présent. Dans ce cas, l'effet recherché est celle « ... d'un présent qui cherche à actualiser pour le lecteur ce qu'il sait pourtant être de l'ordre du passé, procédé fréquent dans les récits historiques: « Nous sommes en telle année... ». », souligne Reuter (2009, p. 72). Effectivement, le roman commence avec: « Août 1987. Un après-midi chaud et étouffant... » (9). Cette tentative montre justement (et nous le verrons plus loin) qu'il existe un autre 
niveau de narration où la narratrice hétérodiégétique évoque un temps révolu (les évènements du roman). Ces deux temps alternent et s'interpénètrent successivement dès le début de l'histoire, de sorte qu'ils semblent former, par moments, une perception unifiante; cela exprime également l'incapacité d'une unification totale. Lors d'un des premiers dialogues avec l'infirmière de la clinique, l'écriture montre cet état d'esprit de la narratrice:

[...]-Lieu de naissance?-Téhéran. Le doux murmure d'une ville lointaine s'éveille lentement dans ma tête et le jardin de Shemiran, comme un songe vert, s'étend derrière mes paupières. Téhéran avec son $r$ qui roule sur le bout de la langue et son a long et profond en finale, m'enveloppe dans son souffle parfumé et m'avale comme la bouche épicée d'un bazar (p. 11).

La perception intériorisée du temps dans laquelle la narratrice semble être immergée alternativement, et les « états de conscience »d'ordre émotionnels perçus « l'un dans l'autre » par elle rappellent l'idée de « durée » qui est selon Bergson:

La durée toute pure est la forme que prend la succession de nos états de conscience quand notre moi se laisse vivre, quand il s'abstient d'établir une séparation entre l'état présent et les états antérieurs. Il n'a pas besoin, pour cela, de s'absorber tout entier dans la sensation ou l'idée qui passe, car alors, au contraire, il cesserait de durer. Il n'a pas besoin non plus d'oublier les états antérieurs: il suffit qu'en se rappelant ces états il ne les juxtapose pas à l'état actuel comme un point à un autre point, mais les organise avec lui, comme il arrive quand nous nous rappelons, fondues pour ainsi dire ensemble, les notes d'une mélodie (1984, p. 67).

Les sons et les images qui «s'éveillent» dans l'esprit de la narratrice, les verbes «s'envelopper » et « avaler » dénotent une immersion totale et incontrôlée des états de conscience de la narratrice dans son passé. L'évocation d'un autre temps et un autre lieu (Téhéran) sous forme de dialogue montre le glissement de la narratrice dans la durée «continue et indivisible » autre qu'un temps « scientifique » et chronologique. En effet, c'est dans la durée que les événements semblent s'interpénétrer de façon continue et hétérogène. La narratrice semble revivre, pour ainsi dire, des moments de son passé dans le présent, au déclic d'un son ou d'une sensation, mais de façon discontinue. «C'est justement cette continuité indivisible de changement qui constitue la durée vraie », précise Bergson. ${ }^{4}$ Aussi la durée, qui représente une temporalité «pure » (non chronologique et donc sans spatialisation), semble former « un tout » intériorisé révélant l'être profond de la narratrice pour qui chaque image, chaque son du présent, fait vivre intuitivement son enfance en Iran dans ses plus infimes détails. Cette façon de « vivre » ou de revivre quasi-instantanément les souvenirs, par l'appropriation des sentiments et des sensations les plus intimes du moi profond sans les dénaturer, est ce que Bergson appelle vivre la durée.

Or, comment la durée peut-elle être combinée avec la nostalgie? La question est de savoir s'il peut y avoir de durée (même parasitée) à partir du moment où il y a nostalgie, ou la nostalgie exclut-elle complètement la durée?

${ }^{4}$ Bergson (1987, p. 166). 


\section{La durée et la nostalgie au présent}

L'histoire comprend donc deux temps vécus en durée par la narratrice. En évoquant les souvenirs de sa mère, cette dernière fait aussi « irruption » dans le présent:

Quelqu'un m'appelle au loin, au-delà des mers et des montagnes. Ma mère arrose les pots de géranium tout autour de la terrasse. C'est la nuit [...] (p. 11).

Aussi le regard sur l'ordre temporel du récit, c'est-à-dire l'emploi du présent dans le cas de l'analepse, dévoile un sens clef dans la lecture de l'œuvre. En effet, en reprenant ici les concepts du temps de l'histoire par rapport au temps du récit, nous observons une « quasi-simultanéité » de ces deux temps. Alors que la narratrice est dans une clinique en France, ses souvenirs lointains de l'Iran s'incarnent dans le présent comme si les souvenirs faisaient partie intégrante du présent de la narration. Nous observons deux faits:

1. L'état dépressif et nostalgique de la narratrice ayant une notion du temps déréglé.

2. Une appréhension spontanée et intuitive de la temporalité, faisant abstraction ou presque de toute délimitation spatio-temporelle. Donc une situation de vécu en durée.

À première vue, le premier phénomène semble engendrer le second. La cause de la perception en durée semble d'abord être l'état dépressif. Est-ce la nostalgie destructrice qui cause un dérèglement temporel et crée une pseudo-durée, ou bien au contraire la durée, comme perception temporelle et moyen profond de création, estelle perturbée et parasitée par la nostalgie?

Nous essaierons d'éclaircir ces problématiques à l'aide d'un autre concept bergsonien en rapport sine qua non avec la durée. Il s'agit de liberté et de son rapport avec la création. Or, sachant que la dépression est plutôt un obstacle négatif, pathologique et parasitaire pour la notion de durée, comment la narratrice pourraitelle s'approprier la liberté afin de créer? En effet, elle sait que pour pouvoir écrire elle doit se libérer de la dépression. C'est-à-dire, en fait, libérer la durée des jougs de la nostalgie parasitaire. Elle dit:

Comment écrire? Par où commencer? Depuis deux ans, cette profonde dépression a absorbé toutes mes pensées. Je suis incapable d'écrire... (p. 19).

Pour Bergson, qui n'est pas un platonicien, la durée n'est pas un état statique, une idée immuable. Alain Panéro ${ }^{5}$ dit à propos de la pensée bergsonienne:

Habituellement, on croit que Bergson nous demande seulement de nous déprendre de l'espace et de vivre la durée réelle; ce qui laisse penser que le dernier mot du bergsonisme est de nous livrer à une intuition aveugle de la durée pure, à une nuit où toutes les vaches sont noires. En vérité, il importe de noter que le bergsonisme reste un rationalisme qui nous demande surtout de penser en durée. Penser en durée, ce n'est pas seulement vivre l'intuition de la

\footnotetext{
5 Panéro (2005) Matière et esprit chez Bergson. (Site consulté en juin 2011: http://pedagogie.ac-amiens. fr/philosophie/PAF/bergson-panero.htm\#r8).
} 
durée. Là encore, il y a une méconnaissance du bergsonisme et un risque réel de contresens. Aux yeux de Bergson, il ne s'agit pas seulement de vivre le temps, il faut aussi tenter de dire, de penser, de communiquer notre expérience métaphysique du temps.

Si la durée bergsonienne n'est pas une « intuition aveugle », elle représenterait plutôt une vision basée essentiellement sur la raison et l'intellect. C'est-à-dire qu'ontologiquement c'est une manière d'être, une vision du monde. Dans ce sens, d'après l'analyse de la tradition des affects (« la joie, la pitié, la colère, la crainte, etc. ») elle n'est pas « contenue » ou anéantie par la nostalgie qui est en fin de compte un affect. ${ }^{6}$ Dans le récit, il s'agit bien d'un affect pathologiquement amplifié qui perturbe la vision en durée de la narratrice, mais qui reste néanmoins contenu dans la vision ontologiquement artistique et créatrice en durée. Libérée de la nostalgie par l'écriture, la narratrice expose son envie d'écrire et de «communiquer » son expérience.

Dire, penser et communiquer la durée est ce qui la rattache à l'acte créateur. Il s'agit ici de l'écriture. Donc l'expérience de la durée se réalise à partir du moment où la narratrice se met à écrire et à créer (par une mise en abyme) le roman de son expérience de la nostalgie. Cet effort de penser la durée introduit la notion de mouvement dans la pensée de Bergson. Celui-ci s'oppose justement à l'idée d'une durée statique et à l'immuable. Dire la durée par l'écriture c'est en même temps la créer et la rendre possible dans l'acte-même de la création. C'est cet acte d'écriture qui rend aussi possible le passage de la nostalgie destructive à la nostalgie réparatrice en tant que mouvement. Aussi vivre la durée ne devient possible qu'en mouvement, c'est-à-dire simultanément à sa création par l'écriture en tant que la forme de son expression. La durée est une première fois «vécue » de façon parasitée (par la dépression) par la narratrice et une deuxième fois exprimée et écrite par le narrateur hétérodiégétique.

Le narrateur hétérodiégétique exprime un temps de narration futur " dissimulé » derrière le présent de la narration du héros-narrateur. C'est au début puis à la fin du roman que la narration laisse un autre indice à l'existence d'un temps futur et du narrateur hétérodiégétique:

J'entends le portail se fermer derrière mon dos et la clinique de Ville-d'Avray, avec ses illusions et ses hallucinations, avec ses gens, ses frayeurs et ses infirmiers, se transforme en une courte nouvelle dans mon esprit, une nouvelle que j'écrirai un jour (pp. 203-204).

« [...] un jour » est le temps post-hospitalisation. De ce fait, le lecteur perçoit d'abord le point de vue du héros-narrateur malade, ensuite celui du narrateur hétérodiégétique. Ces différentes narrations appartiennent cependant à la même personne mais dans des temps différents. L'évocation de la date («Août 1987 ») dès le début de la narration introduit bien l'idée qu'il s'agit en « réalité » d'une narration par analepse. En fait l'auteur signale un temps révolu tout en jouant sur l'ambiguïté

\footnotetext{
${ }^{6}$ Moser (1999), Ibid. p.83.
} 
et la confusion d'une narration instantanée au présent. Pourquoi l'auteur procède-telle à ce choix?

L'évocation de la date « Août 1987 » marque la distance temporelle entre le récit écrit et le récit raconté par la narratrice au présent. Elle révèle l'existence d'un temps post-événementiel. C'est-à-dire la preuve que la narratrice a pu finalement se débarrasser de la nostalgie destructrice, retrouver ses capacités d'écriture et passer à la phase de nostalgie réparatrice. C'est aussi le temps de la vie et le post-deuil d'exil. Bref, il s'agit d'un « futur » qui, au moment de la maladie, fut incertain voire même refusé par la narratrice:

Il me faut parler, regarder, trouver mon moi présent et me projeter dans le futur. C'est au-dessus de mes forces. L'avenir me terrorise, seul le passé est doué de réalité, lui seul a une logique indubitable (p. 16).

Par la suite pourtant, un futur se dessine en filigrane, résultat d'une thérapie, de l'écriture, mais aussi comme une perspective post-exil. Celle qui parle en disant: « Le plaisir d'écrire ranime mon esprit malade. De leurs fils colorés, je tisse un tapis volant qui m'emporte vers le passé le plus lointain, vers les allées boisées du jardin de Shemiran... » (21), c'est précisément ce moi-profond qui finit par se retrouver. Par la logique de la guérison de la protagoniste à la fin du roman, viennent aussi et surtout l'acceptation du présent et la vie du futur:

Un jour seulement un jour comme aujourd'hui, si plein de songes et de promesses, vaut une vie entière. Peut-être que cette heure d'ivresse n'est qu'un moment passager. Peu importe, j'emporte son souvenir avec moi et grâce à la mémoire perpétuelle de ce jour, j'enlumine tous les jours qui sont à venir (p. 205).

La simultanéité spatio-temporelle a donc une signification et une fonction précises. Le narrateur souligne ainsi la gravité et la réalité de cet état nostalgique destructif de la protagoniste qu'elle fut jadis. L'emploi du présent historique est une technique d'écriture créant l'effet d'une focalisation interne à travers laquelle le lecteur «vit» l'enfermement et l'étouffement de la narratrice dans son exil en France. L'autre fonction de cette simultanéité temporelle renvoie en fait à la nécessité de sa création. En effet, c'est par cette voie que l'auteur introduit et exprime la durée dans le récit en même temps qu'elle la crée. La vraie durée c'est donc le processus de la création de l'auteur elle-même, c'est-à-dire le roman écrit par une immersion presque complète dans le passé afin de souligner (et ressentir) au mieux l'essence de ces moments. De la sorte, également pour Bergson, la création artistique et la durée ne sont pas séparables, mais ne font qu'un:

Mais, pour l'artiste qui crée une image en la tirant du fond de son âme, le temps n'est plus un accessoire. Ce n'est pas un intervalle qu'on puisse allonger ou raccourcir sans en modifier le contenu. La durée de son travail fait partie intégrante de son travail (p. 198).

Le lecteur observe alors comment le récit raconté par la narratrice est altéré par la nostalgie et la dépression (ainsi que la durée, d'où la difficulté de l'écriture et de création au début). Seul, l'acte de l'écriture (et son processus) est et raconte la 
durée, en la rendant possible. C'est seulement lors de ce processus, que le moi créateur se libère de la nostalgie destructrice, puisqu'elle la raconte. C'est cette distance prise par l'écriture qui «purifie » la durée de la nostalgie mélancolique. Elle raconte ainsi l'histoire de son moi prisonnier du passé, comme temps cristallisé, mais aussi de l'espace de ce passé sublimé. Car la nostalgie est aussi une affaire d'espace. Le passé vers lequel la narratrice tend est un espace concret mais aussi un cadre symbolique reflétant les bases identitaires. La manière de raconter l'espace, ses détails et sa composition, ouvre la psychologie du personnage. La nostalgie est aussi une projection de l'espace de l'enfance sur le présent de l'adulte en France.

\section{Nostalgie familiale et l'espace}

La mémoire et les souvenirs évoquent un espace perdu et idéalisé. L'espace de l'enfance est souvent synonyme des racines identitaires. Son évocation idéalisée accentue la nostalgie de la narratrice adulte. La nostalgie renvoie à l'espace du pays natal, l'espace où l'identité avait des racines: celui de l'enfance; car si l'exil cause d'abord un malaise et une crise identitaire, c'est dans cet espace que la narratrice cherche à la retrouver.

L'espace fonctionne selon deux perspectives intimement liées. Il est le lieu concret contenant des objets avec leurs bagages émotionnels (la maison, le paysage, les statues du jardin, le bassin...), mais c'est aussi un espace de vie partagé par des personnages, des odeurs et des affects qui racontent la construction identitaire de la narratrice. C'est avant tout l'espace iranien et celle d'une époque historique, d'une mentalité et d'une génération (la bourgeoisie sous Reza Shah Pahlavi). ${ }^{7}$

L'espace et les environs de la maison de Shemiran (éloignée de la ville, au pied de la montagne) reflètent de manière concrète la psychologie de la petite fille originale et insolite:

Mais, moi, j'aime cette maison isolée et je n'ai pas peur de son grand réservoir d'eau, de son bassin plein de grenouilles ou des ombres noires de ses arbres qui ressemblent aux gens méchants (p. 23).

La maison somptueuse, symbole de grandeur familiale, est régie au rythme patriarcal du père solitaire, pilier de tout. Le père définit l'espace, et l'espace le reflète à son tour:

Le jardin de Shemiran est envahi par des statues d'animaux. Au pied des marches menant à la terrasse, sont assis deux lions, immenses, avec la gueule ouverte, prêts à avaler les invités. [...] La partie nord de la pièce est la place privilégiée de père (p. 168).

Toute l'identité, l'imagerie spatiale de la narratrice et son avenir semblent orientés par le vecteur paternel. Le goût et la passion pour l'écriture lui sont aussi transmis par le père: «Il est écrivain et son bureau me fascine. » dit l'enfant (18), et plus loin:

\footnotetext{
7 Reza Khan Mir Panj (1878-1944), est connu sous le nom de Reza Shah Pahlavi. Il régna comme le Chah d'Iran de 1925 à 1941.
} 
«Ce jour-là j'ai décidé que je serai écrivain » (19). Le père devient le symbole du pays natal et de la maison familiale construite par lui, mais aussi le pilier de tout un univers pyramidal, hiérarchique et familial englobant l'identité de la narratrice. La grande famille entoure et protège l'enfant: « [...] comme l'enfant cachée sous le tchador fleuri de sa grand-mère, je me sens à l'abri de tout. » (16). Protection, construction mais aussi l'envahissement et un certain étouffement caractérisent cet espace familial et paternaliste.

L'ombre du père s'étend, en effet, partout. Les enfants guettent leur délivrance de la sieste obligatoire quand «Le moment heureux de la liberté est annoncé par l'ouverture des paupières de père », et si la tranche de la pastèque du serviteur de la maison n'est pas bonne alors: «La punition de père est l'amende salariale. Tout le monde dans la maison reçoit un salaire de père[...] » (169).

L'espace identitaire du passé est tout d'abord représenté par l'image d'un père puissant, stable et protecteur. Dans le texte, la première voix « entendue ", sous forme de discours rapporté, est celle du père, cela souligne son importance ontologique autant que son pouvoir envahissant dans l'univers de la narratrice: « - Je suis d'acier et l'acier ne rouille jamais, disait père » (9). Cela introduit l'actualité et la présence du père chez la narratrice. Il est ensuite ainsi évoqué: "Moi, déclare père, je veux une maison avec jardin [...]». Plus loin, la narratrice expose clairement sa vision idéalisée du père: « Je m'accroche à son image, à sa puissance magique, à la force de l'homme de fer, le vainqueur de la maladie et l'ennemi des faibles. » (10). L'évocation de cette affirmation hyperbolique par la narratrice adulte dénote une certaine auto-ironie. Aussi l'envie d'une certaine émancipation par rapport à l'espace patriarcal et hégémonique se dessine, même si, dans un premier temps, la narratrice légitime et cherche cette identité du passé, à défaut d'accepter le présent et de reconstruire une «nouvelle » identité dans un espace nouveau. Selon Judith Stern, la reconstruction identitaire de l'individu exilé doit dépasser cette " période transitoire pendant laquelle le sentiment d'identité est mis à l'épreuve et en conséquence réveille des sentiments nostalgiques pour la période pendant laquelle cette identité était assurée. ${ }^{8}$ Subséquemment, le passé qui enlise la narratrice par son espace envahissant, la pousse en même temps vers une émancipation et dépassement de soi. L'homme « de fer » et «d'acier» s'éteint un jour et la petite fille doit faire face à la réalité.

Transformations et chocs subis par la petite fille sont allégoriquement reflétés à travers l'espace (dans le récit). Avec la mort du père, c'est toute une époque, tout un espace (la maison, le quartier) qui change:

Le gouvernement a mis en place un nouveau plan d'urbanisme qui relie par une autoroute les deux extrémités de la ville. Cette voie impériale traverse la maison de Shemiran en son milieu (p. 191).

Symbole de la modernité, l'autoroute éventrant la maison en plein milieu marque le début d'une ère nouvelle. C'est le symbole d'une rupture radicale avec une époque, le père et l'identité même de la narratrice. La nostalgie et la solitude sont aussi les

\footnotetext{
${ }^{8}$ Stern (2008, p. 21) (site consulté le 11 novembre 2009. http://rsmq.cam.org/filigrane/archives/nostalg. htm).
} 
conséquences de «l'arrachement » du père de l'espace patriarcal et protecteur, sur quoi reposait l'identité communautaire de la narratrice. En effet, le père symbolise une ère révolue et donc déjà « l'exil » pour l'enfant sans père. En visitant son père malade, elle dit: "Je vais le voir et je déteste ces visites. J'ai honte, comme si la maladie de l'acier était un événement honteux » (187), alors que le père continue de défier et de narguer la maladie et la mort jusqu'au bout avec une philosophie et poésie orientale dans l'esprit et le regard. Ayant perdu un œil il déclare: «Cet unique œil me suffit. Je vois mieux que vous tous. L'œil le plus important se trouve dans le cœur et dans la tête de l'homme. L'œil de l'intelligence, l'œil de la justice. » (189). La maladie et la mort du père emportent avec lui tout un espace de référence identitaire et traditionnel: «Cette mort n'est pas comme les autres morts; c'est la rouille de l'acier, l'achèvement d'une époque et la ruine des habitudes anciennes. » (184). C'est tout un regard, une philosophie, une poésie et une force de vie, mais aussi une domination patriarcale qui disparaît avec le père, créant une sensation de solitude et de vide chez la narratrice déjà exilée, « déterritorialisée » selon le concept développé par Deleuze et Guattari. ${ }^{9}$

De l'autre coté, l'espace de l'exil en France semble aussi vide de toute chaleur, de sens et de réalité. Le ciel pluvieux comme thème typique de différence et de rejet, dans cette littérature, se pose face à la chaleur de l'Iran: « Je vais mourir en exil, sous la pluie incessante, sous un ciel sans soleil. »(10). La guérison s'accompagne d'une reterritorialisation spatiale de l'exil, c'est-à-dire une réappropriation de l'espace en tant que sens. La possibilité d'un nouvel espace de vie et de création se dessine progressivement. Donc, la vision pessimiste évolue à la fin du roman, se reflétant à travers les objets et l'espace:

Je mets mes écrits dans l'armoire et je la ferme à clef. Je jette la clef dans un tiroir et tourne le dos à la mémoire de mon passé. Je veux m'insérer dans l'aujourd'hui, je veux sentir le présent familier des choses qui m'entourent, penser à cette journée ensoleillée, à l'arbre encore frêle devant ma fenêtre, à mes mains qui doucement et patiemment, tournent les pages d'un livre et à mon être en paix avec le monde extérieur (p. 199).

Finalement, il semblerait que l'espace identitaire de la protagoniste, qui s'était construit sur l'univers du père, la maison et la communauté familiale, glisse graduellement vers un ailleurs et se déterritorialise en quelque sorte. L'exil devient cet espace de recréation rendant possible un avenir pour un Je identitaire mutilé.

\section{La schizophrénie et la nostalgie}

L'auteur introduit dans son roman des notions d'ordre physique et psychologique reconnaissables dans le cadre de l'exil chez son personnage. L'état schizophrénique

\footnotetext{
${ }^{9}$ Les termes de « déterritorialisation » et de « reterritorialisation » ont été créés par Deleuze et Guattari dans leur trilogie sur le «Capitalisme et schizophrénie »: Anti-Edipe, Mille Plateaux, et Qu'est-ce que la philosophie?
} 
fait partie de ces notions. Selon la définition de l'Encyclopédie Médicale, ${ }^{10}$ la schizophrénie est: « une psychose, qui se manifeste par la désintégration de la personnalité, et par la perte du contact avec la réalité. » Des exilés d'autres origines ont exprimé cet état psychologique perturbant dû à cette condition. ${ }^{11}$ Le fait d'être dans le pays d'accueil et de vivre en même temps dans le passé du pays natal, constitue et contribue à accentuer un tel état. Dans le cas de la narratrice, la tradition et la culture propagée par l'entourage de l'enfant contribuent fortement au développement de cet état. Les rapports sociaux et la hiérarchie des valeurs poussent l'enfant à être témoin des « contradictions » chez des adultes, dans leurs rapports, face à la vérité et la sincérité. La petite fille observe:

Malgré les mensonges de ma mère, moi, mon frère et mes cousins, nous savons tous que cet oncle invisible est malade, et si les grandes personnes nous demandaient notre avis à nous, les enfants, nous leur répondrions que cet oncle est foutu, qu'il ne guérira certainement pas. Bien entendu, une gifle retentissante nous attendrait pour avoir exprimé nos idées sincèrement (p. 43).

L'enfant observe et comprend beaucoup plus que ne le croient les adultes. Elle voit tous ces mensonges: «Certains soirs ma mère a soudainement mal aux dents et doit aller, tout de suite, au cabinet du Dr Kossari. Le djinn chuchote alors dans mes oreilles que ma mère est une menteuse. » Et un peu plus loin: «Le djinn, à la vitesse du vent, vient à ma rencontre, murmurant que père est cruel et menteur. »(52) Et à la suite de quoi l'enfant rajoute: «Lors des cours d'éducation religieuse, la maîtresse d'école nous dit:-Celui ou celle qui ne respecte pas et n'écoute pas son père et sa mère brûlera dans les feux de l'enfer. »

L'exil et le cadre de vie dans l'enfance ont des effets similaires sur la protagoniste. Les deux suscitent un dédoublement de l'identité. L'on constate, d'un côté, les contradictions d'ordre culturel en France et de l'autre, les lacunes éducatives en Iran. La nostalgie exprime avant tout un manque, un questionnement qui perdure depuis l'enfance, car irrésolu et insatisfait. La nostalgie envers le chauffeur du bus par exemple, ou la «Petite Amie » exprimant des frustrations de l'enfant et leur prolongation en exil. Contrastes et contradictions sont donc d'abord au cœur de la société. En conséquence, l'enfant sensible développe tout un monde intérieur contrastant avec l'extérieur. La schizophrénie commence déjà dans le pays natal et c'est avant tout le signe d'un décalage identitaire derrière une vision critique de la culture.

L'imagination débordante, qui peut être un phénomène naturel chez l'enfant, s'amplifie démesurément. Elle entend les djinns et développe une relation fusionnelle avec la «Petite Amie »: «Je sens qu'un événement étrange s'est produit à l'intérieur de mon corps, je suis devenue la Petite Amie. » (72). D'un côté la «Petite Amie » est une camarade « réelle », extérieure à elle, et de l'autre côté, chef de rébellion, d'émancipation et d'anarchisme, elle symbolise cette inspiration à

\footnotetext{
${ }_{10}$ http://www.doctissimo.fr/html/sante/encyclopedie/sa_1013_schizophrenie.htm.

11 La sociologue iranienne C. Chafiq-Beski évoque l'étude faite sur la situation des réfugiés latinoaméricains par Anna Vasquez et Angela Xavier de Brito, en précisant que « la première étape de l'exil par le traumatisme de départ, deuil et culpabilité, qui contribue à mettre sur pied une situation pratiquement schizophrénique. » (Ibid. p. 24).
} 
la liberté de la petite narratrice elle-même. Une transgression qui doit être «payée » par la peur et la culpabilité. Ayant enfreint les règles et restée en cachette dans les couloirs vides et sombres de l'école, la petite narratrice dit:

Nous déambulons dans les salles de classe et faisons tout ce que nous voulons. Mais en réalité, tout au fond de moi, je suis effrayée par cette liberté, j'ai l'impression qu'un œil invisible est posé sur moi et je retiens mes désirs ainsi que ma volonté (p. 75).

Au fond, la « Petite Amie » devient, par cette petite pirouette, symbole du désir de liberté, d'émancipation et de transcendance des règles et des valeurs, engendrant du même coup l'angoisse de la double identité. L'identité plurielle qui transcende les règles et les interdits dans l'enfance (due aux paradoxes dans l'éducation et la société), réapparaît chez la narratrice adulte exilée sous forme d'un moi schizophrénique partagé entre le présent et le passé, l'atmosphère du pays d'accueil et l'Iran.

Tous ces arrachements aux valeurs affectives (la «Petite Amie », le père, la famille, la maison) contribuent à la perturbation identitaire de la narratrice. La schizophrénie ici a deux faces: d'une part elle représente la fragmentation du moi identitaire (très typique chez les exilés et apatrides en général) qui se morfond dans la nostalgie destructrice, et d'autre part sous les apparences de la « Petite Amie », elle dénote et symbolise la rébellion, et la transgression des espaces et des mœurs interdits. Cet aspect du dédoublement de la personnalité est ambigu car il signale également le potentiel imaginatif de la narratrice comme artiste créatrice. L'arrachement à l'Iran, contient en soi le germe d'un dépassement. Le déracinement est un moment éminent de déterritorialisation psychologique et physique (des normes et des valeurs culturelles et de l'espace). L'exil est un cadre à dépasser; la perturbation qu'il provoque implique chez la narratrice une tentative de reterritorialisation: une réattribution de sens à soi et à son nouvel environnement. C'est en donnant la place qui convient à la réalité actuelle et aux souvenirs que l'identité mutilée se redéfinit. L'évolution de la pensée et de l'identité réside dans cette obligation de redéfinition. C'est en acceptant le présent de l'exil et la vie du pays d'accueil que le moi créateur s'unifie. Enfin c'est à travers l'écriture, et d'intense introspection, que la narratrice procède au travail de deuil et le dépassement du passé. Vaincre la nostalgie (l'enfance et la situation de l'exil) a pour effet le dépassement d'une crise, d'une mutilation identitaire. Sans l'état nostalgique destructif, le temps intérieur est canalisé autrement, c'est-à-dire non pas vers la mort (car le moi prisonnier du passé se meurt), mais vers la vie et la création-vers la durée.

\section{Vers une reconstitution}

La réflexion sur la nostalgie en exil, sa nature et son expérience personnelle dans le roman de Taraghi nous mène inévitablement à considérer la question du temps et de l'espace. Malgré les particularités psychologiques et les expériences personnelles de la protagoniste, le rapport de beaucoup d'autres artistes exilés avec la nostalgie et 
son évolution montre des similarités. Car les conditions et les difficultés constituant le cadre de l'exil sont dans les grandes lignes les mêmes pour la plupart des artistes expatriés. Les problèmes de création, le choc identitaire et culturel restent toujours centraux pour ces derniers.

La nostalgie destructrice représente des caractéristiques vécues similairement par d'autres exilés ${ }^{12}$ : un regard passéiste, une idéalisation du pays natal et une identité mutilée. La notion de durée concrétise, en mettant formidablement en perspective le pouvoir de l'écriture, l'expérience formidable de l'émergence d'une œuvre sur la transcendance de l'état nostalgique et dépressif. L'écriture thérapeutique comme une nécessité de survie, ensuite l'écriture qui pense et écrit la durée comme création artistique. Le lecteur tient en main la deuxième alors que la diégèse parle de la première.

Penser en durée, c'est d'abord laisser vivre son moi profond en toute liberté. L'affiliation pathétique du moi profond au passé est due à la nostalgie destructrice qui obstrue la création et la liberté nécessaire à la création. C'est à force de persévérer dans l'écriture que la narratrice parvient à chasser la nostalgie destructrice et à lui substituer la réparatrice. La nostalgie destructrice est ainsi susceptible de se transformer, mais cela suppose un travail sur les souvenirs et les bases culturelles et éducatives incrustées dans son être. Ainsi, lorsque les fantômes du passé surgissent la nuit: «Souvent, au milieu de la nuit, je me réveille et je vois un visage apparaître [...]. Il est si vivant et si réel que je peux sentir ses doigts invisibles frôler ma peau » (22), le travail de l'écriture les convertit simultanément en personnage de roman par «l'écrivain qui renaît » en même temps. La renaissance du moi créateur par l'écriture. L'acte de reterritorialisation des vestiges culturels du passé, dans un nouveau cadre culturel. En effet, c'est au pays d'accueil, qu'a lieu la mutation identitaire qui se projette dans l'avenir. L'état nostalgique n'est donc pas essentiellement immuable, mais susceptible de changement et d'évolution vers un dialogue culturel. Car il s'agit bien ici d'une phase transitoire où la narratrice fait le deuil d'une des phases de l'exil pour rentrer dans une phase d'interculturalité.

Open Access This article is distributed under the terms of the Creative Commons Attribution Noncommercial License which permits any noncommercial use, distribution, and reproduction in any medium, provided the original author(s) and source are credited.

\section{References}

Bergson, H. (1984). Essai sur les données immédiates de la conscience (4th ed.). Paris: PUF. 67.

Bergson, H. (1987). La perception du changement (1911), repris dans La pensée et le mouvant. Paris: PUF, coll. Quadrige. 166.

Bolzinger, A. (2007). Histoire de la nostalgie entre médecine et psychanalyse. Paris: Campagne première. Chafiq-Beski, C. (2005). L'exil au féminin. Respublica, Autour de l'Exil, 41, 24.

Deleuze, G., \& Guattari, F. (1972). Anti-CEdipe. Paris: Les Editions de Minuit.

Deleuze, G., \& Guattari, F. (1980). Mille plateaux. Paris: Les Editions de Minuit.

Deleuze, G., \& Guattari, F. (1991). Qu'est-ce que la philosophie? Paris: Les Editions de Minuit.

Grisoni, D. (1987). Les abîmes de l'âme. Magazine littéraire, 244, 16.

Karimi Hakkak, A. (2006). Conférence littéraire, Meskoob, S. USA: Kanoone Iranian.

Moser, W. (1999). Mélancolie et nostalgie: affects de la Spätzeit. Etudes littéraires, 31(2), 83-89.

12 Que l'on retrouve dans d'autres œuvres, notamment chez Ghassemi Reza. 
Ortiz, F. (2002). Contrapunteo cubano del tabaco y el azúcar. Madrid: Enrico Mario Santi. Panéro, A. (4 février 2005). Matière et esprit chez Bergson. Site consulté en juin 2011. Reutier, Y. (2009). Introduction à l'analyse du roman. Paris: Armand Colin. 72.

Stern, J. (2008). L'immigration, la nostalgie, le deuil. Revue de psychanalyse filigrane, 5, 21. Taraghi, G. (2003). La maison de Shemiran. Paris: Actes Sud. 